\title{
Anti-inflammatory and antioxidant nanostructured cellulose membranes loaded with phenolic-based ionic liquids for cutaneous application
}

\author{
Eduarda S. Morais ${ }^{a}$, Nuno H.C.S. Silva ${ }^{a}$, Tânia E. Sintra ${ }^{a}$, Sónia A.O. Santos ${ }^{a}$, \\ Bruno Miguel Neves ${ }^{b}$, Isabel F. Almeida ${ }^{c}$, Paulo C. Costa ${ }^{c}$, Inês Correia-Sá ${ }^{d}$, Sónia P.M. Ventura ${ }^{a}$, \\ Armando J.D. Silvestre ${ }^{a}$, Mara G. Freire ${ }^{a}$, Carmen S.R. Freire ${ }^{a, *}$ \\ ${ }^{a}$ CICECO, Department of Chemistry, University of Aveiro, 3810-193 Aveiro, Portugal \\ ${ }^{\mathrm{b}}$ Department of Medical Sciences and Institute of Biomedicine - iBiMED, University of Aveiro, 3810-193 Aveiro, Portugal \\ ${ }^{\mathrm{c}}$ UCIBIO-REQUIMTE, Laboratory of Pharmaceutical Technology, Department of Drug Sciences, Faculty of Pharmacy, University of Porto, $4051-401$ Porto, Portugal \\ ${ }^{\mathrm{d}}$ Department of Plastic, Aesthetic, Reconstructive and Aesthetic Surgery, Centro Hospitalar de S. João, 4200-319 Porto, Portugal
}

\section{A R T I C L E I N F O}

\section{Keywords:}

Bacterial cellulose

Ionic liquids

Phenolic acids

Antioxidant properties

Antiinflammatory properties

Skin treatment

Topical applications

\begin{abstract}
A B S T R A C T
The utilization of natural compounds, such as phenolic acids and biopolymers, in the healthcare domain is gaining increasing attention. In this study, bacterial nanocellulose (BC) membranes were loaded with ionic liquids (ILs) based on phenolic acids. These ionic compounds, with improved solubility and bioavailability, were prepared by combining the cholinium cation with anions derived from caffeic, ellagic and gallic acids. The obtained BC-ILs membranes were homogeneous, conformable and their swelling ability agreed with the solubility of each IL. These membranes revealed a controlled ILs dissolution rate in the wet state and high antioxidant activity. In vitro assays performed with Raw 264.7 macrophages and HaCaT keratinocytes revealed that these novel BC-ILs membranes are non-cytotoxic and present relevant anti-inflammatory properties. Diffusion studies with Hanson vertical diffusion cells showed a prolonged release profile of the ILs from the BC membranes. Thus, this work, successfully demonstrates the potential of BC-ILs membranes for skin treatment.
\end{abstract}

\section{Introduction}

The demand for innovative and high-performance skin healthcare materials and products, including wound healing membranes, and products to protect skin from adverse effects is increasing considerably. Skin diseases, accidents and chirurgical interventions are also widely associated with skin damage and injuries (Dai \& Mumper, 2010). Furthermore, some of these systems can have dermocosmetic applications to prevent premature skin aging, and cancer associated to sun exposure (Dai \& Mumper, 2010; Podda \& Grundmann-Kollmann, 2001), which due to UV-induced formation of noxious reactive oxygen species (ROS) (Podda \& Grundmann-Kollmann, 2001), can lead to wide variety of pathological effects, such as DNA damage, lipid peroxidation, as well as the activation of inflammatory pathways (Guidi et al., 2006; Kovacic \& Somanathan, 2013; Massaad, 2011; Sas, Robotka, Toldi, \& Vécsei, 2007; Yu \& Anderson, 1997).

Bacterial cellulose (BC) is a type of cellulose produced by non- pathogenic bacteria that is characterized by a 3-D structure with an ultrafine network of cellulose nanofibers (Czaja, Krystynowicz, Bielecki, \& Brown, 2006). Because of this peculiar ultrastructure and unique properties, namely biocompatibility (Chawla, Bajaj, Survase, \& Singhal, 2009), high water absorption capability, in situ moldability and mechanical properties (Jones, Currie, \& Martin, 2002), BC is an ideal material for several biomedical applications, including transdermal drug delivery patches, wound healing membranes and skin substitutes, as well as cosmetic products (Czaja et al., 2006). However, native BC membranes lack for important functionalities, namely antioxidant and anti-inflammatory properties that are of extreme relevance on skin protection and healing (Czaja et al., 2007; Drury \& Mooney, 2003; Fontana et al., 1990; Jones et al., 2002; Mayall, Mayall, Mayall, Rocha, \& Marques, 1990; Nguyen, Gidley, \& Dykes, 2008; Schirhagl, 2014). In fact, it is assumed that $\mathrm{BC}$ has poor influence on the biochemical state of chronic wounds that persist in the inflammatory phase of the normal healing process and therefore fail to heal (Wiegand, Elsner, Hipler, \&

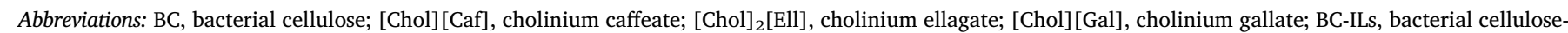

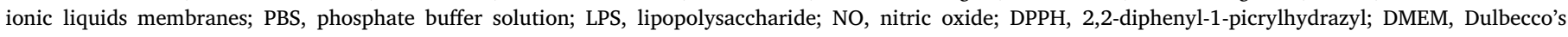
Modified Eagle's Medium; FBS, fetal bovine serum

* Corresponding author.

E-mail address: cfreire@ua.pt (C.S.R. Freire).
} 
Klemm, 2006). However, only a couple of studies reported strategies to improve the positive features of $\mathrm{BC}$ as wound dressing. Wiegand et al. (2006) focused on the formation of a composite of BC with collagen type I that is able to reduce the proteases amount, interleukin concentration and the ROS activity. Additionally, Barud et al. (Barud et al., 2013) demonstrated the considerable antimicrobial activity and wound healing capability of a BC membrane containing an ethanol soluble propolis extract. In a different study, it was also demonstrated that $\mathrm{BC}$ including vaccarin increases wound healing by improvement of cell adhesion (Qiu, Qiu, Cui, \& Wei, 2016).

The use of natural bioactive compounds, as phenolic compounds (natural antioxidants) in different fields, including biomedical, cosmetic and pharmaceutical areas, have shown a considerable growth in the past decades (Mandal, Yadav, Yadav, \& Nema, 2009). However, due to their instability, associated with photodegradation in the presence of oxygen, keeping constant their activities in formulations during their claimed shelf life, is often problematic. Moreover, these natural bioactive compounds typically display low solubility in water, as well as a low bioavailability. Thus, chemical modification aiming at increasing their stability and solubility in aqueous media, while not raising their cytotoxicity, can be seen as a promising approach for their safe and effective use in topical formulations (Kusumawati \& Gunawan, 2013). The combination of active pharmaceutical ingredients (APIs) with ionic liquids (ILs) constitutes a solution to tackle the mentioned challenge since it can synergistically enhance the antioxidant activity of those compounds as well as their solubility and bioavailability (Marrucho, Branco, \& Rebelo, 2014). ILs are salts, usually composed of large organic cations and organic or inorganic anions, without an ordered crystalline structure, and thus tend to be liquid at temperatures lower than conventional high-melting inorganic salts (Plechkova \& Seddon, 2008; Rogers \& Seddon, 2003). Combining phenolic acids as anions with the cholinium cation to form ILs constitute an interesting alternative since these compounds have higher water-solubility, antioxidant activity, and anti-inflammatory properties, and similar toxicity levels when compared with their acidic counterparts (Sintra et al., 2015). Due to their outstanding properties, these compounds can be incorporated into different materials, like BC, for topical applications.

In this context, the aim of this work was to produce biobased nanostructured materials with antioxidant and anti-inflammatory properties for topical applications (e.g. chronic wound healing), by incorporating phenolic-based ILs with these features, such as cholinium ellagate $\left([\mathrm{Chol}]_{2}[\mathrm{Ell}]\right)$, cholinium gallate $([\mathrm{Chol}][\mathrm{Gal}])$ and cholinium caffeate ([Chol][Caf]) into bacterial cellulose membranes. The absorption process of ILs into the BC membranes and their release profiles were investigated and optimized. The different BC-ILs membranes were characterized in what concerns their morphology, thermal stability and mechanical properties, using Fourier transform infrared spectroscopy with attenuated total reflection (FTIR-ATR), solid state nuclear magnetic resonance (NMR), X-ray diffraction (XRD), scanning electron microscopy (SEM), thermogravimetry (TGA) and mechanical (tensile) tests. Finally, the evaluation of their biological properties and in vitro cytotoxicity was carried out and their permeation profile through epidermal membranes was evaluated using Hanson vertical diffusion cells.

\section{Materials and methods}

\subsection{Chemicals}

Cholinium hydroxide, [Chol] OH (in methanol solution at $45 \mathrm{wt} \%$, Sigma-Aldrich, St. Louis, MO, USA) and phenolic acids, namely gallic acid (99.5 wt\% purity, Merck, Kenilworth, New Jersey), ellagic acid (97 wt\% purity, Alfa Aesar, Lancashire, United Kingdom) and caffeic acid ( $99 \mathrm{wt} \%$ purity, ACROS, Geel, Belgium) were used in the synthesis of ILs. 2,2-Diphenyl-2-picrylhydrazyl hydrate (DPPH) was acquired from Sigma-Aldrich (St. Louis, MO, USA) and methanol (HPLC grade) from VWR (Radnor, New Jersey). All other reagents were of analytical grade (Sigma-Aldrich, St. Louis, MO, USA).

Lipopolyssacharide (LPS) from Escherichia coli (serotype 026:B6), penicillin and streptomycin were obtained from Sigma Chemical Co. (St. Louis, MO, USA). Dulbecco's Modified Eagle's Medium (DMEM), fetal bovine serum (FBS), 2',7'-dichlorodihydrofluorescein diacetate (DCFH-DA) and Hoechst were obtained from Fisher Scientific (Leicestershire, UK).

\subsection{Synthesis and characterization of phenolic-based ILs}

Three cholinium-based ILs were synthesized by the neutralization of [Chol] $\mathrm{OH}$ with the respective phenolic acid, namely gallic, caffeic and ellagic acids, as reported elsewhere (Sintra et al., 2015). Briefly, [Chol] $\mathrm{OH}$ was added dropwise to gallic and caffeic acids solution in methanol, 1.1 equivalent, at $0{ }^{\circ} \mathrm{C}$, under nitrogen atmosphere. Regarding the $[\mathrm{Chol}]_{2}[\mathrm{Ell}]$ synthesis, the $[\mathrm{Chol}] \mathrm{OH}$ was added to the ellagic acid solution in methanol, with a molar ratio of $2: 1$, due to the presence of two acidic groups with similar pKa values. The reaction mixtures were stirred overnight at room temperature, under nitrogen atmosphere and protected from light, producing each IL and water as byproduct. Methanol and water were then removed under reduced pressure. In the synthesis of [Chol][Caf], the unreacted acid was eliminated by washing the solid with acetone $(3 \times 20 \mathrm{~mL})$, followed by filtration to remove the phenolic-based IL. The same procedure was adopted for [Chol][Gal] yet replacing acetone by methanol. Finally, the residual solvent was removed under reduced pressure and the obtained compounds were dried under high vacuum for at least $48 \mathrm{~h}$.

The synthesized ILs were analyzed by ${ }^{1} \mathrm{H}$ and ${ }^{13} \mathrm{C}$ NMR showing a high purity level (Supporting information - Figs. S1-S3). ${ }^{1} \mathrm{H}$ and ${ }^{13} \mathrm{C}$ NMR spectra were recorded using a Bruker Avance 300 (France) at $300.13 \mathrm{MHz}$ and $75.47 \mathrm{MHz}$, respectively, using deuterated water/dimethyl sulfoxide $\left(\mathrm{D}_{2} \mathrm{O} / \mathrm{DMSO}\right)$ as solvent and trimethylsilyl propanoic acid/tetramethylsilane (TSP/TMS) as internal reference. The full names, acronyms, and chemical structures of the studied ILs are depicted in Fig. S4 - Supporting information.

\subsection{BC production}

Pure BC membranes ( $~ 99 \mathrm{wt} \%$ water content) were produced using the bacterial strain Gluconacetobacter sacchari (Trovatti, Serafim, Freire, Silvestre, \& Neto, 2011) and following a previously described procedure (Hestrin \& Schramm, 1953). After growing for 6 days, BC membranes were removed from culture media, washed three times with $0.5 \mathrm{M}$ $\mathrm{NaOH}$ at $80^{\circ} \mathrm{C}$ for $30 \mathrm{~min}$, and then thoroughly washed with distilled water until neutral $\mathrm{pH}$. Pure $\mathrm{BC}$ membranes were kept in distilled water in a sterile environment at $4{ }^{\circ} \mathrm{C}$ until use (Trovatti, Silva et al., 2011).

\subsection{Preparation and characterization of BC-IL membranes}

Wet BC membranes, with $2.0 \pm 0.1 \mathrm{~cm}$ diameter and about $0.8 \pm 0.1 \mathrm{~cm}$ thickness were weighted (around $23 \mathrm{mg}$ of dry $\mathrm{BC}$ ) and circa $50 \%$ (estimated by weight loss) of their water content was drained by hand-pressing the biomaterial between two acrylic plates at room temperature. BC membranes were then soaked in $1 \mathrm{~mL}$ of aqueous solutions containing [Chol][Caf], [Chol $]_{2}[\mathrm{Ell}]$ or $[\mathrm{Chol}][\mathrm{Gal}]$ with a concentration of $10 \mathrm{mg} \mathrm{mL}^{-1}$. After the complete absorption of the solution by the BC membranes, the BC-IL membranes were placed over Petri dishes and dried at room temperature under a nitrogen atmosphere. Dried BC-IL membranes were kept in a desiccator until use. Different sized membranes were latter prepared for different analysis, i.e. mechanical and permeation assays.

FTIR-ATR spectra of BC and BC-IL dried membranes (as well as of the individual components) were obtained on a Perkin Elmer spectrometer (USA) equipped with a single horizontal Golden Gate ATR cell. Thirty-two scans were acquired in the $4000-600 \mathrm{~cm}^{-1}$ range, with a resolution of $4 \mathrm{~cm}^{-1}$. 
The surface of the BC and BC-IL membranes was analyzed by SEM by cutting an adequate size membrane sample, while for cross-section images the membranes were broken after immersing them in liquid nitrogen. The samples were then covered with carbon and analyzed using a Hitachi SU-70 microscope (Japan) at 4 and $10 \mathrm{kV}$. The same procedure was also made with membranes after dissolution assays that were dried afterwards.

${ }^{13} \mathrm{C}$ solid-state cross-polarized magic-angle spinning nuclear magnetic resonance $\left({ }^{13} \mathrm{C}\right.$ CP-MAS NMR) spectra were recorded on a Bruker Avance 400 spectrometer (France). Samples (BC and BC-ILs) were packed into a zirconia rotor sealed with Kel-F caps and spun at $7 \mathrm{kHz}$. The acquisition parameters were as follows: $4 \mu \mathrm{s} 90^{\circ}$ pulse width, $2 \mathrm{~ms}$ contact time, and $4 \mathrm{~s}$ dead time delay.

Tensile assays were performed using an Instron 5944 (USA) testing machine with Bluehill 3 software in tensile mode with a $1 \mathrm{kN}$ load cell. Samples were strips of $70 \mathrm{~mm} \times 5 \mathrm{~mm}$ and the gauge length $30 \mathrm{~mm}$. At least 7 strips were tested for each sample. The corresponding stress (MPa)-strain (\%) curves were plotted, and the Young's modulus values were determined from the slope of the low strain region near $0.05 \%$. Results were expressed as the average \pm SD.

The decomposition temperatures of the membranes were determined by TGA conducted with a Shimadzu TGA 50 analyzer (France) equipped with a platinum cell. Samples were heated at a constant rate of $10^{\circ} \mathrm{C} \mathrm{min}{ }^{-1}$, from room temperature to $800{ }^{\circ} \mathrm{C}$, under a nitrogen flow of $20 \mathrm{~mL} \mathrm{~min}^{-1}$. The thermal decomposition temperatures were taken as the onset of significant $(\geq 0.5 \%)$ weight loss, after the initial moisture loss. Results were expressed as the average temperature $\pm \mathrm{SD}$.

X-ray diffraction patterns were determined using a Phillips X'pert MPD diffractometer (Netherlands) using $\mathrm{Cu} \mathrm{Ka}$ radiation. The XRD measurements were performed with a scan step size of $0.02^{\circ}$ and a time per step of $2.5 \mathrm{~s}$ from 4 to $402 \theta$ range.

Crystallinity index (C.I.) and percentage crystallinity (\% Crystalline) of BC samples were calculated as follows (Erbas Kiziltas, Kiziltas, Blumentritt, \& Gardner, 2015):

C. $I=\frac{I_{200}-I_{a m}}{I_{200}}$

$\%$ Crystalline $=\frac{I_{200}}{I_{200}+I_{a m}} \times 100$

where $I_{200}$ is the maximum intensity of diffraction of the (200) lattice peak $\left(2 \theta\right.$ of $\left.22^{\circ}-23^{\circ}\right)$ and $I_{a m}$ is that of the amorphous part between $2 \theta$ of $18^{\circ}$ and $19^{\circ}$, where the intensity is minimum.

Triplicates were made for all the aforementioned characterization techniques.

\subsection{Swelling rate assays}

Swelling rate assays were conducted using dried BC and BC-IL membranes. Membranes were weighted and then soaked in individual containers with distilled water or PBS buffer ( $\mathrm{pH} \mathrm{7.4)}$ at room temperature, during $24 \mathrm{~h}$. At different times, samples were taken out of media, the excess of solvent was gently removed with absorbent paper and membranes were weighted and re-immersed again. Triplicates were conducted for all samples, and results were expressed as the average \pm SD. The swelling of the membranes was calculated according to Eq. (3):

$\frac{w_{w e t}-w_{d r y}}{w_{d r y}} \times 100$

where $\mathrm{w}_{\mathrm{dry}}$ and $\mathrm{w}_{\text {wet }}$ are the weight of dried and wet BC samples, respectively.

The thickness and width of the membranes (before and after immersion in the swelling media) were measured using a digital micrometer (model MDE-25TJ, Mitutoyo Corp., Tokyo, Japan). The mean thickness and width were calculated from three measurements taken at different locations in each film sample, and results were expressed as the average \pm standard deviation (SD).

\subsection{Dissolution assays}

BC-IL dried membranes were placed in a closed flask containing $180 \mathrm{~mL}$ of phosphate buffer solution (PBS) at $\mathrm{pH} 7.4$ to approach the blood $\mathrm{pH}$, under magnetic stirring and protected from light, at $37^{\circ} \mathrm{C}$. The cholinium-based ILs release was then evaluated. At determined time intervals (during $24 \mathrm{~h}$ ), $1 \mathrm{~mL}$ of solution was withdrawn, and the same volume of fresh buffer was added to maintain a constant volume. The cholinium-based salt content in each aliquot was determined by UV spectroscopy, as described below. Triplicates were conducted for all samples and PBS solution was used as reference for the baseline correction of the values, and results were expressed as the average \pm standard deviation (SD). The IL content at each time was plotted as a cumulated percentage release, determined according to Eq. (4):

$\frac{1 \times C_{n-1}}{180}+C_{n}$

where $\mathrm{C}_{n-1}$ and $\mathrm{C}_{n}$ are the IL concentrations at time $n-1$ and $n$. Two replicates were performed for each sample.

Dissolution assays of the ILs in methanol were also performed with the same time intervals in order to relate the amount of IL dissolved in the media with its antioxidant activity. The quantification of choliniumbased salts in the solutions resulting from the dissolution assays was carried out by UV spectroscopy, using a Thermo Scientific Evolution 600 spectrophotometer (USA). The wavelengths selected were $286 \mathrm{~nm}$ for [Chol] [Caf], $253 \mathrm{~nm}$ for [Chol] ${ }_{2}$ [Ell] and $259 \mathrm{~nm}$ for [Chol] [Gal] as described elsewhere (Sintra et al., 2015) using calibration curves previously established and presented in Tables S1 and S2 - Supporting information.

The results for dissolution assays with both methanol and PBS buffer were also fitted to several mathematical models for drug release behavior, by doing linear regression using least square approximation (Dubey, Malviya, \& Sharma, 2014).

\subsection{In chemico antioxidant activity assays}

The antioxidant activities of BC-ILs were determined using the 2,2diphenyl-1-picrylhydrazyl (DPPH) radical scavenging assay. The principle of the assay is based on the color change of the DPPH solution from purple to yellow, as the radical is quenched by the antioxidant. Change in color was monitored by visible spectroscopy at $517 \mathrm{~nm}$ (Alam, Bristi, \& Rafiquzzaman, 2013).

Briefly, $3.34 \mathrm{~mL}$ of a DPPH solution $(1 \mathrm{mM})$ in methanol was mixed with $50 \mathrm{~mL}$ of methanol with the BC-IL membrane in the medium. The average amount of IL in each membrane used in this work can be found in Supporting information - Table S3. Samples were kept in the dark for $30 \mathrm{~min}, 1 \mathrm{~h}, 2 \mathrm{~h}, 4 \mathrm{~h}, 6 \mathrm{~h}$ and $24 \mathrm{~h}$ at room temperature and then the decrease in the absorbance at $517 \mathrm{~nm}$ was determined (Huang, Ou, \& Prior, 2005). A blank control was made with $250 \mu \mathrm{L}$ of DPPH solution in methanol, and then methanol was added until the volume of $4 \mathrm{~mL}$ was reached. For comparison purposes, the same process was applied to the precursor acids of each IL, using $10 \mu \mathrm{gL}^{-1}$ of acid solution instead of methanol until the volume of $4 \mathrm{~mL}$ was reached. Readings were also carried out with pure ILs in a methanol solution. DPPH radical scavenging activity, AA(\%), was determined according to Eq. (5):

$\mathrm{AA}(\%)=\frac{\left(\mathrm{A}_{0}-\mathrm{A}_{1}\right)}{\mathrm{A}_{0}} \times 100$

where $A_{0}$ is the absorbance of the control and $A_{1}$ is the absorbance of the sample at $517 \mathrm{~nm}$. 


\subsection{Biological assays}

\subsubsection{Cell cultures}

Murine Raw 264.7 macrophages (ATCC number: TIB-71) were cultured in Dulbecco's Eagle Medium (DMEM) containing $4.5 \mathrm{mg} \mathrm{mL}^{-1}$ glucose, $4 \mathrm{mM}$ L-glutamine, $1.5 \mathrm{mgmL}^{-1}$ sodium bicarbonate and supplemented with $10 \%$ non-inactivated fetal bovine serum (FBS), 100 $\mathrm{IU} \mathrm{mL} \mathrm{m}^{-1}$ penicillin, and $100 \mu \mathrm{gL}^{-1}$ streptomycin. The human keratinocyte cell line HaCaT (DKFZ, Heidelberg, Germany) was cultured in same DMEM medium containing heated inactivated FBS. Cells were incubated at $37{ }^{\circ} \mathrm{C}$ in a humidified atmosphere of $95 \%$ of air and $5 \%$ of $\mathrm{CO}_{2}$ and were used after reaching $70-80 \%$ confluence, which occurs approximately every 3 days after each passage.

\subsubsection{Cell viability assays}

In order to investigate the biocompatibility of the $\mathrm{BC}$ matrices containing the different ILs, their effect on macrophage and keratinocyte viability/metabolic activity was assessed by the resazurin assay (Brien, Wilson, Orton, \& Pognan, 2000). Briefly, $1.5 \times 10^{6}$ Raw 264.7 or $1.0 \times 10^{6} \mathrm{HaCaT}$ cells were plated per well of a 6 well plate and let to stabilize overnight. Then, the $\mathrm{BC}$ membranes were put into contact with cell cultures during $24 \mathrm{~h}$ by means of $24 \mathrm{~mm}$ Trasnwell inserts with $0.4 \mu \mathrm{M}$ polycarbonate membranes (Corning, NY, USA). Resazurin was added to cells (final concentration of $50 \mu \mathrm{M}$ ) during the last 2 and $1 \mathrm{~h}(\mathrm{~s})$ of incubation for HaCat and Raw 264.7, respectively. Finally, $200 \mu \mathrm{L}$ from each condition were transferred to a 96 wells plate and the absorbance of resorufin (the product of the resazurin reduction) measured at 570 and $600 \mathrm{~nm}$ in a standard spectrophotometer BioTek Synergy HT (Biotek Instruments, Winooski, VT, USA). The data are the average of two biological independent experiments conducted in duplicate for each condition and the results were expressed as the average cell viability \pm SD. The BC and BC-IL membranes used in this study were previously sterilized at $135^{\circ} \mathrm{C}$.

\subsubsection{In-vitro antioxidant assays}

The antioxidant activity of BC-ILs membranes was addressed by their capacity to prevent LPS-induced oxidative stress in macrophages. Raw cells were plated at $0.05 \times 10^{6}$ per well of a $\mu$-Chamber slide (IBIDI GmbH, Germany), allowed to stabilize overnight and then stimulated with $1 \mu \mathrm{g} / \mathrm{mL}$ LPS during $16 \mathrm{~h}$. Culture medium incubated during $24 \mathrm{~h}$ with each material to be tested was added $1 \mathrm{~h}$ prior to LPS stimulation. At the end of incubation period, cells were washed three times with $\mathrm{HBSS}$ (in mM: $1.3 \mathrm{CaCl}_{2}, 0.5 \mathrm{MgCl}_{2}, 5.3 \mathrm{KCl}, 138 \mathrm{NaCl}, 0.44$ $\mathrm{KH}_{2} \mathrm{PO}_{4}, 4.2 \mathrm{NaHCO}_{3}$, and $0.34 \mathrm{Na}_{2} \mathrm{HPO}_{4}, \mathrm{pH}$ 7.4) and then loaded with $5 \mu \mathrm{M} \mathrm{H} \mathrm{H}_{2}$ DCFDA and $0.5 \mu \mathrm{g} \mathrm{mL}^{-1}$ Hoechst in HBSS for $30 \mathrm{~min}$ at $37^{\circ} \mathrm{C}$ in the dark. Cells were washed three times with HBSS, and analysed with an Axio Observer Z1 fluorescent microscope (Zeiss Group, Oberkochen, Germany) at $63 \times$ magnification.

\subsubsection{Anti-inflammatory assays}

The potential anti-inflammatory activity of the BC containing cholinium-based ILs was tested by analyzing their capacity to inhibit LPSinduced NO production in macrophages. The production of NO was measured by the accumulation of nitrite in the culture supernatants, using a colorimetric reaction with the Griess reagent. The cells were plated at $3 \times 10^{5}$ cells/well in 48-well culture plates, allowed to stabilize for $12 \mathrm{~h}$, and then incubated (as described in 2.8.1) with the culture medium (control), in presence of three membranes with $0.167 \mathrm{mg}$ of [Chol] [Gal], [Chol] [Caf], [Chol] ${ }_{2}$ [Ell] for $24 \mathrm{~h}$ in order to have the concentration of $56 \mu \mathrm{g} \mathrm{mL}^{-1}$ in the media. At the end of the incubation, $100 \mu \mathrm{L}$ of culture supernatants was collected and mixed with equal volume of the Griess reagent $[0.1 \%$ (w/v) N-(1-naphthyl) ethylenediamine dihydrochloride and $1 \%(\mathrm{w} / \mathrm{v})$ sulfanilamide containing $\left.5 \%(\mathrm{w} / \mathrm{v}) \mathrm{H}_{3} \mathrm{PO}_{4}\right]$ during $30 \mathrm{~min}$, in the dark. After $30 \mathrm{~min}$ incubation in the dark, the absorbance at $550 \mathrm{~nm}$ was measured using a standard spectrophotometer BioTek Synergy HT (Biotek Instruments,
Winooski, VT, EUA) (Sintra et al., 2015). Multiple group comparisons were made by One-Way ANOVA analysis, with a Dunnett's Multiple Comparison post-test. Statistical analysis was performed using GraphPad Prism, version 6.01 (GraphPad Software, San Diego, CA, USA). Significance levels are as follows: ${ }^{*} \mathrm{p}<0.05,{ }^{* *} \mathrm{p}<0.01$, $* * * \mathrm{p}<0.001, * * * * \mathrm{p}<0.0001$.

\subsubsection{In-vitro skin permeation assays}

Human abdominal skin samples were obtained from women who were submitted to an abdominoplasty in Centro Hospitalar São João, Portugal, who signed the respective informed consent. Approval of the Ethics Committee of Hospital São João was obtained. After collection, skin samples were transported under refrigerated conditions. Hypodermis was removed using a scalpel (blade $n^{\circ} 24$ ), and then the skin surface was washed, dried using a cotton swab, wrapped in aluminum foil, and frozen at $-20^{\circ} \mathrm{C}$. Prior to the diffusion experiments the skin was defrosted and cut to an appropriate size. Skin biopsies were obtained using a biopsy punch ( $30 \mathrm{~mm}$ diameter). The epidermis was separated by immersing the skin in water at $65^{\circ} \mathrm{C}$ for $80 \mathrm{~s}$, according to previously optimized protocols (Klingman \& Christophel, 1963). Permeation experiments $(n=6)$ with epidermal membranes were conducted on glass Hanson vertical diffusion cells with a receptor volume of $\sim 7 \mathrm{~mL}$ and a diffusional area of $1.77 \mathrm{~cm}^{2}$. The continuously stirred receptor medium was isotonic phosphate buffered saline (PBS, $\mathrm{pH}$ 7.4). The receptor compartment was thermostated to $37^{\circ} \mathrm{C}$. BC-IL membranes with a load of $4.175 \mathrm{mg}$ of IL were cut to a size of $1.77 \mathrm{~cm}^{2}$ that fitted the surface area of the donor compartment and covered the entire epidermal surface. At designated time intervals, $2 \mathrm{~mL}$ of the receptor solution was withdrawn completely from the receptor compartment and immediately replaced with fresh and pre-thermostated PBS (Silva et al., 2013, 2014). Quantitative analysis of the drug was performed by HPLC (Ultimate 3000, Dionex, Germany) with a reverse phase method using a C18 column with a $3 \mu \mathrm{m}$ pore dimension (Teknokroma, Brisa LC2 TR-010499, $10 \times 0.46 \mathrm{~cm}$ ). Mobile phase was methanol:water (70:30) solution, and the flow rate and the injection volume were set at $0.5 \mathrm{~mL} \mathrm{~min}^{-1}$ and $10 \mu \mathrm{L}$, respectively. Results were expressed as the average cumulative permeation \pm SD. Data statistical treatment was made through ANOVA analysis with a confidence interval of $95 \%$.

The results of the permeation assays were fitted to several mathematical models for drug release behavior, by doing linear regression using least square approximation (Dubey et al., 2014).

\section{Results and discussion}

\subsection{Production and characterization of BC-IL membranes}

BC membranes loaded with cholinium-based ILs paired with anions derived from phenolic acids, namely cholinium ellagate ([Chol $\left.]_{2}[\mathrm{Ell}]\right)$, cholinium gallate ([Chol][Gal]) and cholinium caffeate ([Chol][Caf]), were prepared by soaking partially drained $(50 \% \mathrm{w} / \mathrm{w}$ water content) $\mathrm{BC}$ membranes in $10 \mathrm{mg} / \mathrm{mL}$ aqueous solutions of the cholinium-based ILs. $1 \mathrm{~mL}$ of these solutions was added to $4 \mathrm{~cm}^{2}$ membranes to obtain an IL content per membrane of around $10 \mathrm{mg}$ (Table S3 - Supporting information).

Fig. 1 presents the visual aspect of BC-ILs membranes, in which it is possible to observe the homogeneity of the membranes, associated with a good distribution of the ILs within the 3D network of BC. SEM images

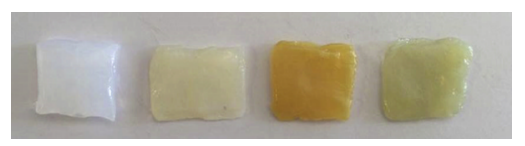

Fig. 1. Macroscopic appearance of the wet $\mathrm{BC}$ membranes incorporating the different ILs. From right to left: BC, BC-[Chol] [Caf], BC-[Chol ${ }_{2}[\mathrm{Ell}], \mathrm{BC}$-[Chol] [Gal]. The produced membranes had a water content of $62.12 \pm 4.40 \%$. 


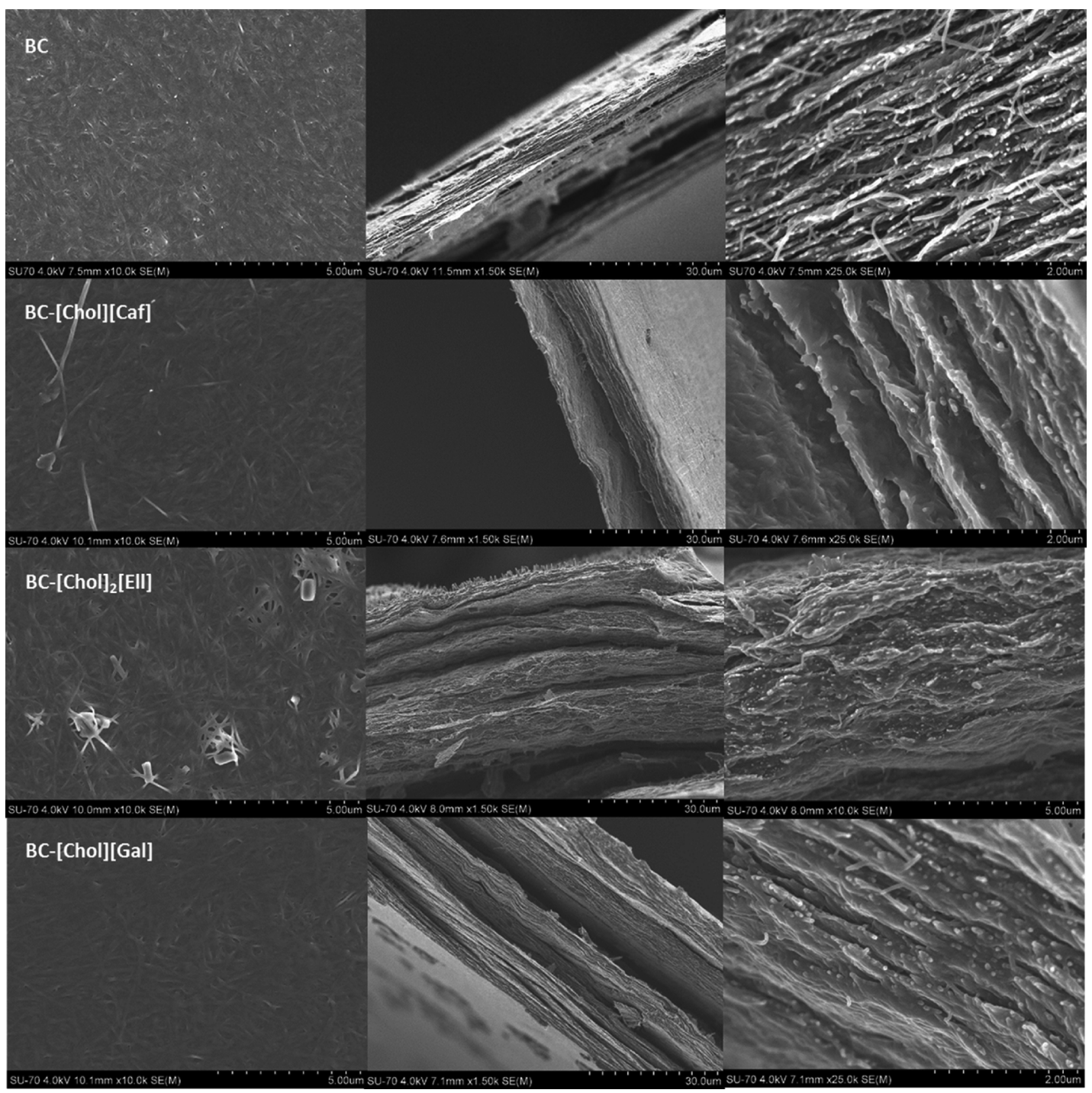

Fig. 2. Surface and transversal SEM images of BC and BC-IL membranes. The first column corresponds to surface images, while the second and third columns correspond to transversal SEM images.

of the surface and cross-section of the membranes with [Chol][Caf], $[\mathrm{Chol}]_{2}[\mathrm{Ell}]$ and [Chol] [Gal] are given in Fig. 2. The typical BC surface tridimensional nanofibrillar network was not affected by the incorporation of the different ILs. A similar trend was observed for the lamellar cross-section morphology that was only filled with the ILs. These results are in agreement with previous works on $\mathrm{BC}$ used as water soluble drug-delivery systems and nanocomposites (Silva et al., 2014, 2013; Trovatti, Silva et al., 2011; Wei, Yang, \& Hong, 2011), in which the spaces between the BC strands were also simply filled with the different drugs or polymers used (Silva et al., 2013). Moreover, the SEM images confirm a uniform dispersion of the ILs in the membranes surface and bulk with no agglomerates formation. Nevertheless, in the case of $[\mathrm{Chol}]_{2}[\mathrm{Ell}]$, there appears to be some IL crystallization on the surface of the membranes, probably due the lower solubility of this IL as well to a lower affinity with cellulose fibrils.

BC-IL membranes, pure ILs, and BC membrane were also analyzed by FTIR (Fig. S5 - Supporting information) in order to further confirm the incorporation of the ILs into the BC matrix. All the spectra were taken in triplicates and using different locations of the samples. The description and identification of the peaks in BC and pure IL spectra can be found in Supporting information. The FTIR spectrum of BC displays the typical peaks of a cellulosic substrate (Cunha et al., 2007; Silva et al., 2014). In general, the spectra of the BC-ILs membranes are a sum of those of BC and pure IL, confirming that the phenolic-based ILs were successfully incorporated into the BC network. However, the spectra of the BC-ILs membranes are much more similar to that of BC since the ILs content is only about $20 \%$ of their total weight. Moreover, some slight peaks deviations can be perceived in the spectra of the BC-[Chol] [Caf] and $\mathrm{BC}-[\mathrm{Chol}][\mathrm{Gal}]$ membranes, particularly in the range of $1250-1750 \mathrm{~cm}^{-1}$, when compared with the spectra of the pure ILs. Specifically, these peaks correspond to the angular bending of $\mathrm{CH}$ $\left(1500 \mathrm{~cm}^{-1}\right)$ and the $\mathrm{C}-\mathrm{OH}$ stretching $\left(1373 \mathrm{~cm}^{-1}\right)$. This suggests that strong interactions (e.g. dipolar interactions and hydrogen bonds) were established between these two ILs and the BC fibrils.

The incorporation of ILs into the BC membranes was also confirmed by solid ${ }^{13} \mathrm{C}$ CPMAS NMR (Fig. 3). The spectrum of $\mathrm{BC}$ is in close agreement with literature (Kono, Yunoki, Shikano, \& Fujiwara, 2002; Tokoh, Takabe, Sugiyama, \& Fujita, 2002). The complete peak identification for BC and BC-ILs can be found in Supporting information. The NMR spectra of the BC-IL membranes show the typical ${ }^{13} \mathrm{C}$ resonances of the ILs and of the $\mathrm{BC}$ membrane, confirming once more that the ILs were successfully incorporated in the BC network. Furthermore, the peaks in $\mathrm{BC}[\mathrm{Chol}][\mathrm{Caf}]$ and $\mathrm{BC}-[\mathrm{Chol}][\mathrm{Gal}]$ spectra also suffer slightly deviations in the ${ }^{13} \mathrm{C}$ chemical shifts when compared to the ones identified in the spectra of the pure ILs. The deviations were identified

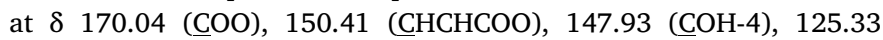



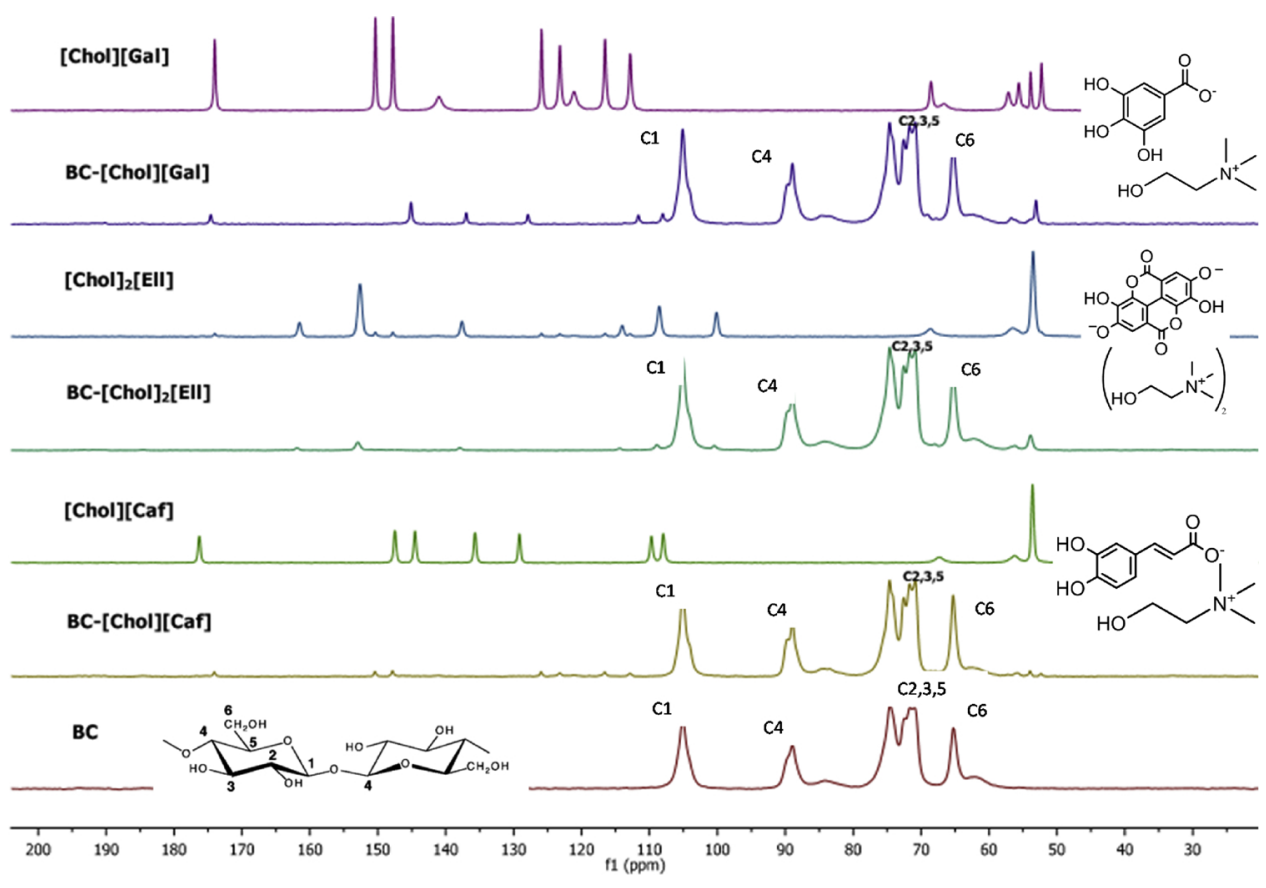

Fig. 3. Solid ${ }^{13} \mathrm{C}$ CPMAS NMR spectra of BC, BC-ILs and pure ILs. Chemical shift in parts per million (ppm).

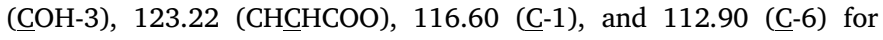
[Chol] [Caf] and $\delta 174.43$ (COO), 145.12 ( $\underline{\mathrm{C}}-3$ and $\underline{\mathrm{C}}-5), 136.99$ (C-4), 127.90 ( $\mathrm{C}-1), 111.65$ ( $\mathrm{C}-2$ and $\underline{\mathrm{C}}-6)$ ), in [Chol] [Gal] in the pure IL spectra. This is a further indication that these two ILs strongly interact with $\mathrm{BC}$ causing the resonances to be shifted, in agreement with the FTIR data. These results are in accordance to the SEM analysis in which $[\mathrm{Chol}]_{2}[$ Ell $]$ seems to be crystallized in the BC membrane surface.

The thermal stability of BC and BC-IL membranes was assessed by TGA (Fig. S6 - Supporting information). BC presents a decomposition temperature close to $360.0^{\circ} \mathrm{C}$, consistent with literature data (Cunha et al., 2007; Mohd Amin, Ahmad, Halib, \& Ahmad, 2012). All ILs loaded $\mathrm{BC}$ membranes are however less thermally stable than $\mathrm{BC}$ because of the ILs lower thermal stability (Sintra et al., 2015). The initial decomposition temperatures of the BC-ILs increase in the following order: $\mathrm{BC}$-[Chol] [Caf] $\quad\left(187.1 \pm 1.0^{\circ} \mathrm{C}\right)<\mathrm{BC}-[\mathrm{Chol}][\mathrm{Gal}] \quad\left(189.2 \pm 1.0^{\circ} \mathrm{C}\right)<\mathrm{BC}-$ $[\mathrm{Chol}]_{2}[\mathrm{Ell}]\left(189.6 \pm 1.0^{\circ} \mathrm{C}\right)$, that is in accordance with the stability of the pure ILs ([Chol] [Caf] $\left(155.0 \pm 1.0^{\circ} \mathrm{C}\right)<[\mathrm{Chol}][\mathrm{Gal}]$ $\left(185.3 \pm 1.0^{\circ} \mathrm{C}\right)<[\mathrm{Chol}]_{2}[\mathrm{Ell}]\left(265.0 \pm 1.0^{\circ} \mathrm{C}\right)($ Sintra et al., 2015)). The first degradation step at $100^{\circ} \mathrm{C}$ in the thermograms (Fig. S6 - Supporting information) corresponds to the evaporation of water, while the second and third ones are attributed to the degradation of the IL-enriched fraction and to the degradation of the $\mathrm{BC}$ enriched fraction, respectively. However, for $\mathrm{BC}-[\mathrm{Chol}][\mathrm{Caf}]$ and $\mathrm{BC}-[\mathrm{Chol}][\mathrm{Gal}]$ the $\mathrm{Td}_{\mathrm{i}}$ is higher than those of pure ILs (155.0 and $185.3^{\circ} \mathrm{C}$, respectively (Sintra et al., 2015)), which might result from the strong interaction between BC and these ILs as pointed out by FTIR, ${ }^{13} \mathrm{C}$ NMR and SEM results. Nonetheless, the decrease on the thermal stability of $\mathrm{BC}$ membranes after incorporation of the ILs does not compromise their application because the sterilization processes are typically carried out at temperatures below $135^{\circ} \mathrm{C}$, posing no risk of degradation of the material.

XRD patterns of the BC-ILs membranes were also acquired in order to understand if there are any changes in the crystalline structure of $\mathrm{BC}$ with the incorporation of ILs. The diffractograms of BC and BC-ILs membranes are shown in Fig. S7 - Supporting information. BC presents the typical cellulose I pattern and the BC-IL membranes preserved most of the XRD pattern of pure BC. The main diffraction signals are observed at around $2 \theta=14.5,16.8,22.6$ and $34.9^{\circ}$, assigned to the 101 , $10 \overline{1}$ (Cunha et al., 2007; Meshitsuka \& Isogai, 1995; Cunha et al., 2007; Meshitsuka \& Isogai, 1995). Additional peaks are also observed in the
BC-IL diffractograms, and correspond to the presence of ILs. The crystallinity and crystallinity index values of the BC membranes (Table S4 Supporting information) only slightly decrease after the incorporation of ILs. Specifically for BC[Chol][Ell] (decrease of 2\%) and BC-[Chol] [Gal] (decrease of 4.5\%) this is expected due to the amorphous parts of the ILs that are present in the BC matrix.

$\mathrm{BC}$ and $\mathrm{BC}-\mathrm{ILs}$ membranes were also characterized regarding their mechanical properties (tensile tests). Three particular parameters were determined, namely elongation at break (\%), Young Modulus (MPa) and tensile strength (MPa) (Fig. 4). The elongation at break of BC[Chol] [Caf] and BC-[Chol] [Gal] membranes is slightly lower than that of pure BC $(2.30 \%)$, with values of $1.93 \%$ and $0.93 \%$, respectively. On the contrary, the $\mathrm{BC}-[\mathrm{Chol}]_{2}[\mathrm{Ell}]$ membrane shows the highest elongation value $(2.86 \%)$. The stronger interactions established between [Chol] [Caf] and [Chol] [Gal] and the cellulose strands, as confirmed by FTIR and NMR, can in part explain this reduction on the elongation at break.

BC membrane presents the highest modulus, viz $17.84 \pm 8.8 \mathrm{GPa}$, which is in accordance with literature (Yamanaka et al., 1989), followed by BC-[Chol][Caf] (14.27 $\pm 9.4 \mathrm{GPa}), \quad$ [Chol][Gal] $(14.09 \pm 4.0 \mathrm{GPa})$ and BC-[Chol $]_{2}[\mathrm{Ell}](11.26 \pm 6.4 \mathrm{GPa})$, which is in accordance with its higher elongation at break and less rigidity, as previously discussed. BC presents also the highest tensile stress $(3.76 \pm 9.3 \mathrm{GPa})$ followed by $\mathrm{BC}-[\mathrm{Chol}][\mathrm{Caf}]$ and $\mathrm{BC}-[\mathrm{Chol}]_{2}[\mathrm{Ell}]$, which presents tensile stress values approximately $30 \%$ lower than BC. $\mathrm{BC}$-[Chol] [Gal] presents the lowest tensile stress, $(0.94 \pm 0.35 \mathrm{GPa})$, and taking into account its lower elongation at break, can be considered the most fragile membrane (Tomé et al., 2015). However, the membranes are still quite malleable as depicted in Fig. S8 - Supporting information. Although some modifications to the main mechanical characteristics of BC were provoked by the incorporation of ILs, they do not compromise the application in skin care treatments.

The results observed for tensile strength of the studied BC-ILs are still superior when compared with other polysaccharides with active compounds incorporation for wound healing applications (Ma et al., 2017). Furthermore, normal skin shows tensile strength in the 2.5-16 MPa range (Silver, 1994) and since our results for tensile strength are quite superior, $\mathrm{BC}$ presents the required characteristics for wound treatment. 

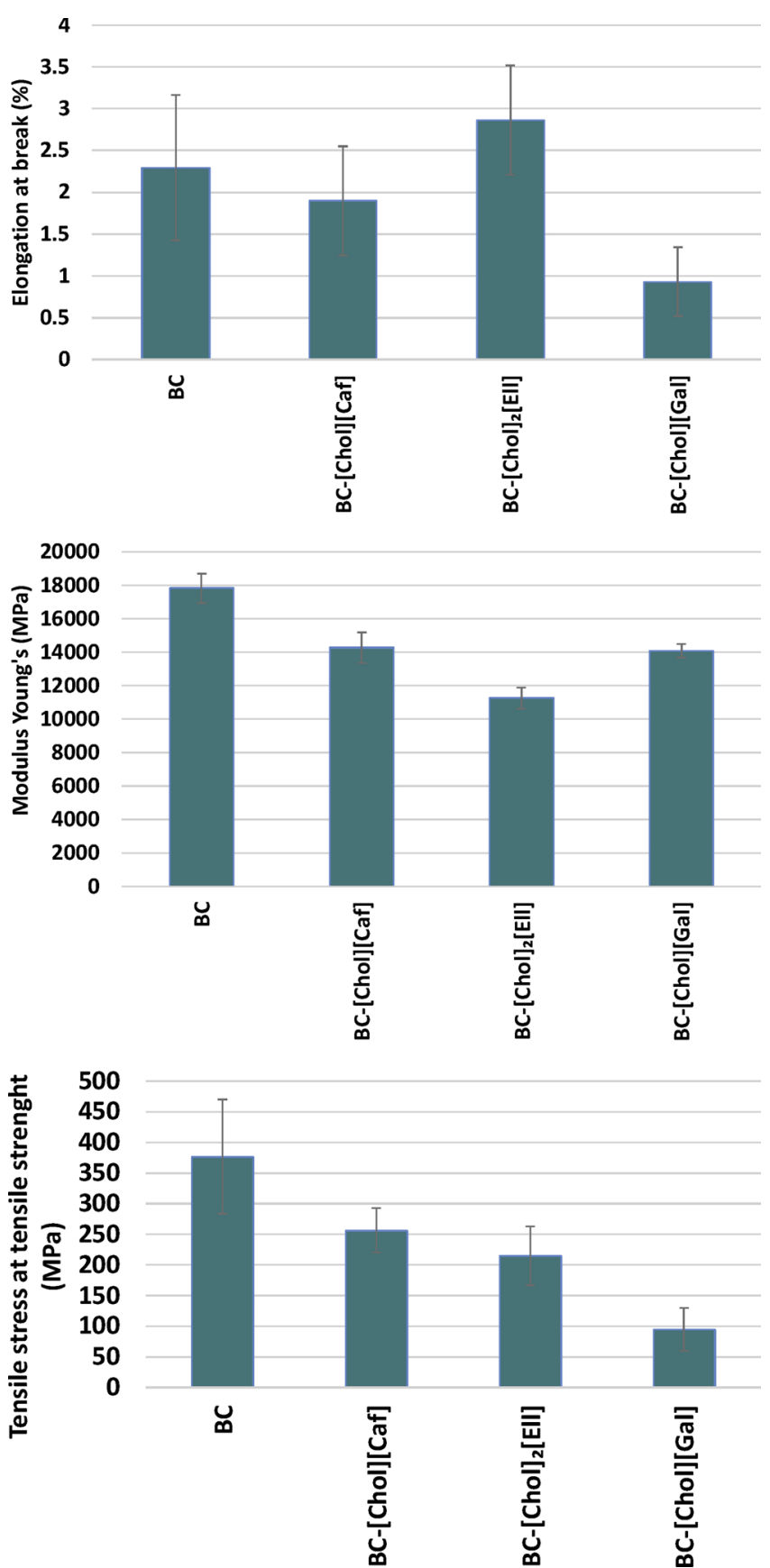

Fig. 4. Elongation at break (\%), Young's modulus (MPa) and Tensile stress at tensile strength (MPa) of BC and BC-ILs (results are expressed as average \pm SD).

\subsection{Swelling rate assays}

The swelling behavior of the obtained BC-IL membranes in water and PBS was investigated because it is an important parameter on topical drug delivery and wound healing, since the ability of re-hydration when in contact with skin influences the releasing rate of the bioactive compounds and the absorption of exudates (Czaja et al., 2006).

All BC-ILs membranes show a fast absorption of water in the first $3 \mathrm{~h}$ of the assay, as shown in Fig. 5, followed by a slight increase until $24 \mathrm{~h}$. These are promising results since the swelling rate plays a major role on the release of ILs from the membrane to the skin. These swelling rates follow the ILs solubility in water ([Chol][Caf] $=2722.47 \pm 63.30$ $\mathrm{mmol} \mathrm{L}^{-1}, \quad[\mathrm{Chol}]_{2}[\mathrm{Ell}]=622.97 \pm 36.98 \mathrm{mmol} \mathrm{L}^{-1}, \quad[\mathrm{Chol}][\mathrm{Gal}=$ $2402.27 \pm 6.09 \mathrm{mmol} \mathrm{L}^{-1}$ ) (Sintra et al., 2015). The swelling rate of
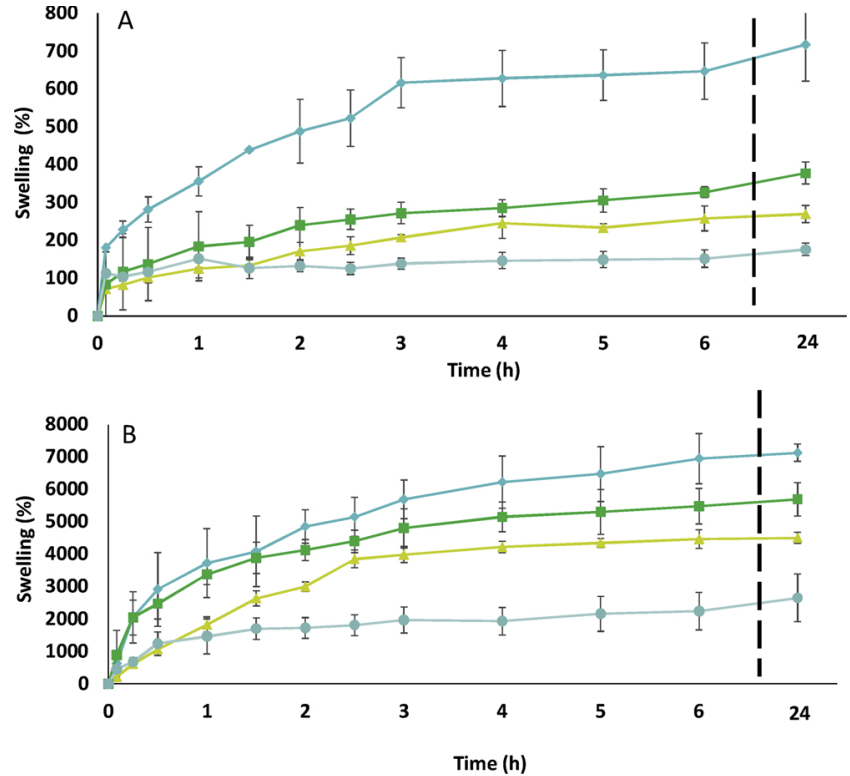

Fig. 5. Swelling rate assays results for BC (๑), BC-[Chol][Caf $](\diamond)$, BC$[\mathrm{Chol}]_{2}[\mathrm{Ell}](\triangle)$ and BC- $[\mathrm{Chol}][\mathrm{Gal}](\square)$. Figure A - Swelling in water; Figure B - Swelling in PBS buffer (results are expressed as average \pm SD).

all BC-ILs is significantly higher than that of BC, which is related to the fact that the ILs are hydrophilic in nature. Additionally, the incorporation of the ILs molecules within the BC 3D structure avoids the structure collapse during drying and therefore a reduction of the number of hydrogen bonds between the BC fibrils, typically associated with the decrease of the re-hydration ability of $\mathrm{BC}$ after drying (Trovatti, Silva et al., 2011). The swelling behavior of the membranes was also investigated in a PBS buffer solution, and it follows the same trend as the swelling with water, although the swelling percentages are considerably higher. Finally, the thickness and width of the membranes was measured before and after soaking in water and PBS for $24 \mathrm{~h}$, and although no significant changes on width were observed, an increase on the thickness was observed after soaking the membranes in water and in PBS (Supporting information - Figs. S9 and S10).

\subsection{Dissolution assays}

The dissolution assays from the loaded membranes were performed during $24 \mathrm{~h}$ and a PBS buffer solution at $\mathrm{pH} 7.4$ was used in order to mimic the $\mathrm{pH}$ of the human blood. The results of the dissolution in PBS (Fig. 6) show that the dissolution of ILs from the dried BC membranes is always faster (nearly 90\%) than from the wet counterparts, independently of the IL used. This behavior can be explained due to the possibility of having the ILs concentrated at the surface as shown by the SEM images of BC-[Chol $]_{2}[$ Ell $]$. Furthermore, dry BC membranes are much thinner when compared with wet BC-IL membranes, and thus there is a faster diffusion of the ILs present in the BC membrane matrix. It was previously established that the tested ILs have a higher solubility in water than the respective acids (Sintra et al., 2015), which supports their quick dissolution into the PBS buffer aqueous solution.

According to Fig. 6, for dry BC-[Chol][Caf] and BC-[Chol][Gal] membranes, the dissolution is instantaneous, reaching maximum dissolution rates of $92.03 \pm 0.85 \%$ and $100.44 \pm 1.58 \%$, respectively. In the case of BC-[Chol $]_{2}[$ Ell $]$ the dissolution profile was quite different and a lower dissolution rate $(44.06 \pm 0.99 \%)$ was observed. On the other hand, for both BC-[Chol][Caf] and BC-[Chol][Gal] wet membranes, only about $70 \%$ dissolution of the ILs content in membranes was reached. This may be due to the pKa difference between the different acidic precursors of the phenolic-based ILs, resulting in the protonated state of $[\mathrm{Chol}]_{2}[\mathrm{Ell}]$ in the PBS solution which in turn will 

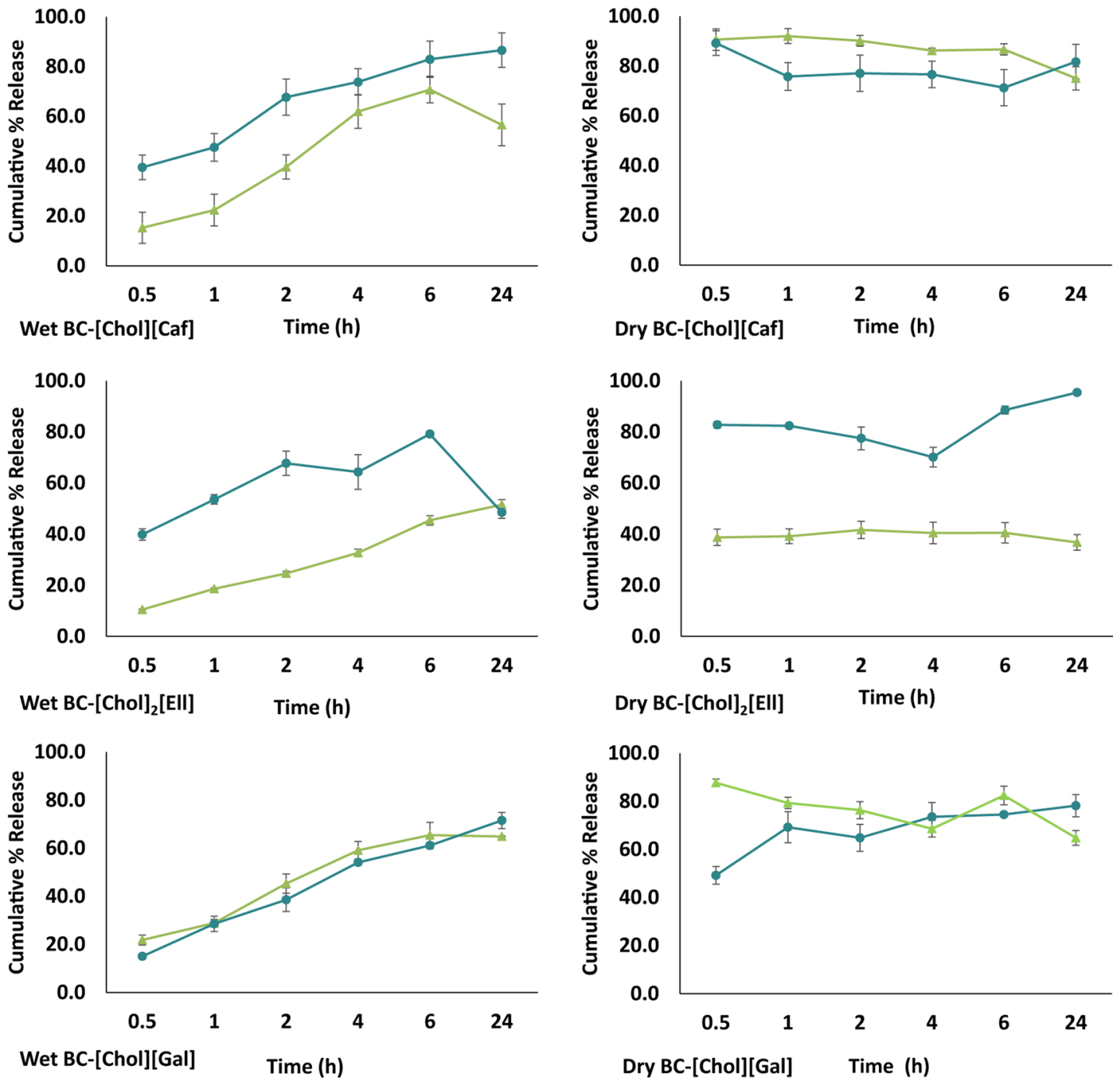

Fig. 6. Cumulative percentage release of IL into the PBS buffer solution ( $\triangle$ ) and methanol (O) (results are expressed as average \pm SD).

result in lower solubility. The higher dissolution percentages were observed at $8 \mathrm{~h}$ with an IL release of $73.91 \pm 0.94 \%$ and $65.95 \pm 0.05 \%$, respectively. With $\mathrm{BC}-[\mathrm{Chol}]_{2}[\mathrm{Ell}]$, the dissolution from the wet membranes was also much lower reaching only $51.58 \pm 0.97 \%$ at $24 \mathrm{~h}$. These results demonstrate that the dissolution profiles were essentially governed by the solubility of the ILs rather by their interactions with the BC fibrils (Sintra et al., 2015).

By fitting the cumulative percentage dissolution values of BC-ILs wet membranes to the Korsmeyer-Peppas Model, the $\mathrm{n}$ values obtained (0.5) are correspondent to a Fickian diffusion behavior, meaning that BC does not exhibit a barrier to IL diffusion (Dubey et al., 2014). On the other hand, for the dry BC-IL membranes and since the release of the IL to the media is almost instantaneous, it was not possible infer any specific release profile.

Moreover, $[\mathrm{Chol}]_{2}[\mathrm{Ell}]$ has an unsteady behavior since after $2 \mathrm{~h}$ in the buffer aqueous solution a decrease of its amount is already perceived that can in part explain the dissolution profile previously discussed. However, this mechanism should be further studied in order to understand what would be its behavior in the human organism. Finally, the SEM images of the BC membranes taken after the dissolution assays in PBS (Supporting information - Fig. S11) reveal the gaps between the hollow or collapse gaps between the fibbers resulting from the dissolution of the ILs. However, in the case of BC-[Chol $]_{2}[\mathrm{Ell}]$ the IL crystals at the surface are still visible confirming that it was not completely solubilized in the PBS solution.
Fig. 6 presents the dissolution of the ILs from the BC-ILs membranes in methanol. This assay was conducted to correlate the amount of dissolved ILs and the results of the DPPH tests in methanol. The results for methanol vary considerably depending on the IL and on the state of the membrane (wet or dry). Similarly to the dissolution results obtained in PBS, there is a faster dissolution of ILs when using dry membranes, while wet membranes allow a more controlled release of ILs. The wet membranes exhibit a zero order burst behavior, since a large bolus of IL is released until $30 \mathrm{~min}$, and then the release is constant over time (Dubey et al., 2014), as showed by the linearity in the graphs presented in Fig. 6.

However, for both BC-[Chol][Caf] and BC-[Chol][Gal] there seems to be a faster dissolution rate of the ILs in PBS than in methanol when using dry membranes. In the case of wet membranes, the dissolution of [Chol] [Caf] is faster and higher in methanol, while with [Chol] [Gal] it is similar. As for $[\mathrm{Chol}]_{2}[\mathrm{Ell}]$, it shows a higher affinity for methanol than for PBS.

\subsection{In chemico antioxidant activity assays}

In Fig. 7 are depicted the results of the dissolution assays in methanol and the antioxidant activity of the membranes determined using the DPPH test. The antioxidant activity is maintained over $80 \%$ for both BC-[Chol] [Caf] and BC-[Chol] [Gal], either with dry or wet membranes, showing that the amount of IL being released by the membranes was 


\section{BC-[Chol][Caf]}
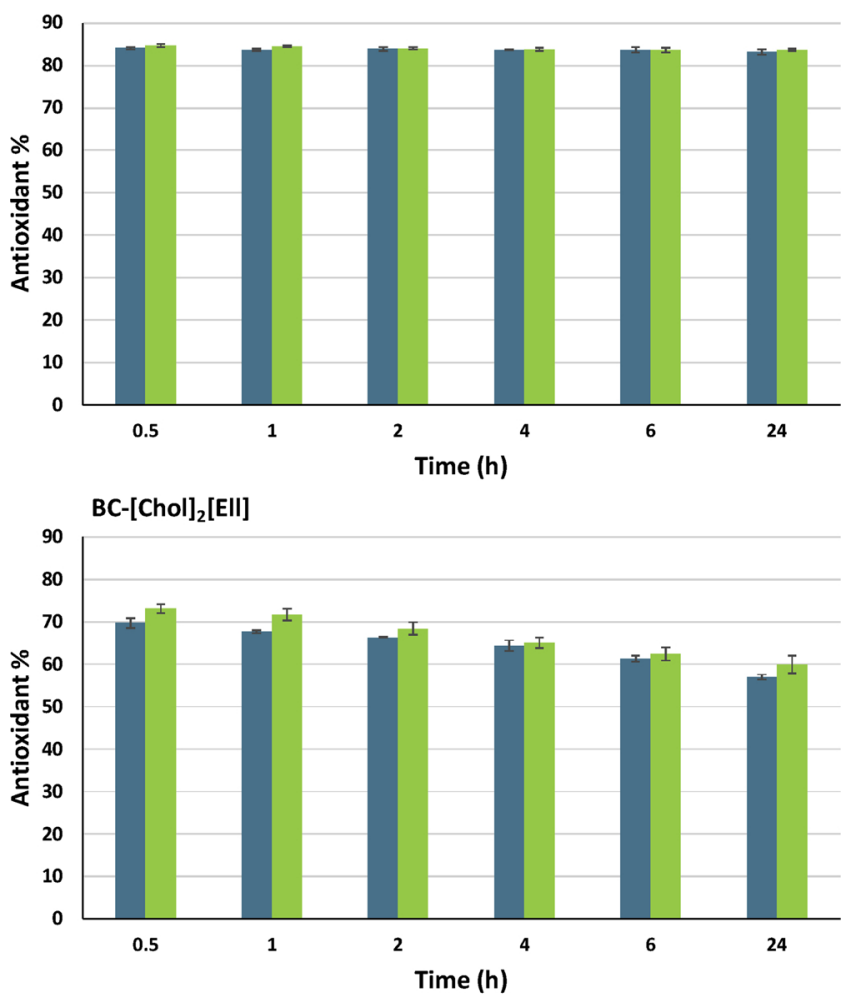

BC-[Chol][Gal]

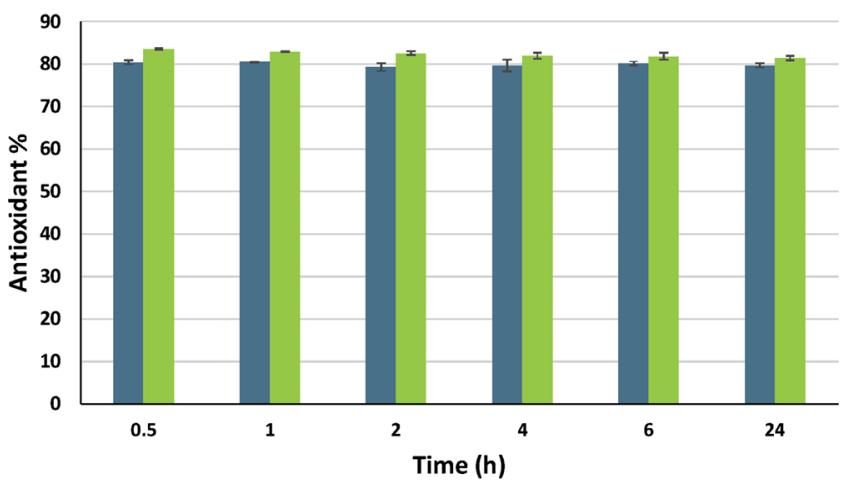

Fig. 7. Antioxidant activity in methanol of dry ( $\square$ ) and wet ( $\square$ ) BC-ILs membranes (results are expressed as average \pm SD).

enough to maintain a higher antioxidant activity. On the other hand, $\mathrm{BC}-[\mathrm{Chol}]_{2}[\mathrm{Ell}]$ has a lower antioxidant activity than the other two studied BC-ILs membranes and suffers a small decrease with time. In general, both wet and dry BC-ILs membranes display similar antioxidant activity results.

The comparison of the antioxidant activity of the synthesized ILs with their acidic precursors incorporated in BC (Supporting information - Fig. S12), show that a smaller quantity of IL (10 mg in BC) leads to the same antioxidant activity of a higher amount of acid ( $37.5 \mathrm{mg}$ of acid in the methanol solution), and similar antioxidant results were obtained using BC-ILs and ILs. In the case of ellagic acid, we could not measure the antioxidant activity due to dissolution problems. The same issue was observed in previous works (Queimada, Mota, Pinho, \& Macedo, 2009; Sintra et al., 2015).

According to the results of the dissolutions and antioxidant activity in solution, and having in mind drug delivery applications, the wet membranes seem to be the most promising since they allow a prolonged diffusion of the active compound.

\subsection{Biological assays}

In order to evaluate if the BC-IL membranes could be used as topical materials with no adverse effects, preliminary cytotoxicity assays were carried out using cells relevant in the skin physiology, namely macrophages and keratinocytes. The average weight of the BC and BC-IL membranes used in the biological assays can be found in the Supporting information - Table S5. The results for the cytotoxicity of BC and BC-ILs are depicted in Figs S13 and S14 - Supporting information. Pure BC membranes and BC-ILs do not cause any decrease in cell viability (above $>90 \%$ ) for both cell lines. These observations are in line with existent reports in literature showing good biocompatibility profiles for either BC (Helenius et al., 2006; Jeong et al., 2010) and choliniumbased ILs (Sintra et al., 2015; Tomé et al., 2015).

in vitro antioxidant assays were performed to validate the results obtained by the DPPH test. As can be seen in Fig. 8, exposure of cells to medium from BC-IL membranes atenuated LPS-induced oxidative stress (green fluorescence) in macrophages. These results are in agreement with thoses obtained in the DPPH antioxidant activity test, with BC[Chol] [Gal] and BC-[Chol][Caf] showing a superior cellular antioxidant capacity.

Anti-inflammatory assays were performed using Raw 264.7 with the goal of confirming that the anti-inflammatory capacity of the ILs was not affected by their incorporation in the BC membranes (Sintra et al., 2015). We observed that exposure of cells to BC-ILs membranes significantly decrease the LPS-induced NO production, indicating a relevant anti-inflammatory potential (Fig. 9). BC-[Chol][Caf] has the best anti-inflammatory activity promoting a significant decrease of $35 \%$ on the production of NO. BC-[Chol] [Gal] and BC-[Chol $]_{2}[\mathrm{Ell}]$ diminish in $23.5 \%$ and $28.7 \%$ the NO production, respectively. These results are in agreement with the anti-inflamatory activity of the percursor phenolic acids (Corbett et al., 2010; Jung et al., 2008; Kroes, van den Berg, Quarles van Ufford, van Dijk, \& Labadie, 1992) and of the corresponding ILs (Sintra et al., 2015). Considering all the biological features evidenced by these membranes, we can conclude that they present enormous potential for application in several clinical situations, namely burned skin and post-surgery, where the reduction of inflamation and skin healing is essential.

\subsection{In-vitro skin permeation assays}

The permeation flux of [Chol][Caf] and [Chol][Gal] from the BC membranes was finally tested in vitro using human epidermal skin in Hanson vertical diffusion cells and using PBS buffer at $37^{\circ} \mathrm{C}$ in order to simulate the conditions of the human skin. BC-[Chol $]_{2}[\mathrm{Ell}]$ was not used in this assay due to precipitation problems. Fig. 10 presents the cumulative permeation values for each hour of the assays. The different parameters calculated are presented in Table S6 - Supporting information. Although the flux $(\mathrm{J})$ seemed higher for [Chol][Gal] $\left(5.42 \mu \mathrm{g} \mathrm{cm}^{-2} \mathrm{~h}^{-1}\right)$ than for [Chol][Caf] $\left(4.93 \mu \mathrm{g} \mathrm{cm}^{-2} \mathrm{~h}^{-1}\right)$, the ANOVA analysis (Table S7 - Supporting information), shows that the difference between these results had no statistical significance $(p=0.43)$. The IL permeation exhibits zero order kinetics with a burst effect in the first hour of the assay, from that point a constant permeation of IL is observed (Dubey et al., 2014). The assays had the duration of $5 \mathrm{~h}$, and the flux and permeated amount showed that a slow and sustained drug release was achieved, corresponding to the major goal of the current work.

\section{Conclusion}

In this work, the possibility of the application of ILs with antioxidant and anti-inflammatory properties to produce bioactive BC membranes for cutaneous application was investigated. It was proven that cholinium-based ILs paired with anions derived from phenolic acids can be successfully incorporated into BC membranes, allowing a 


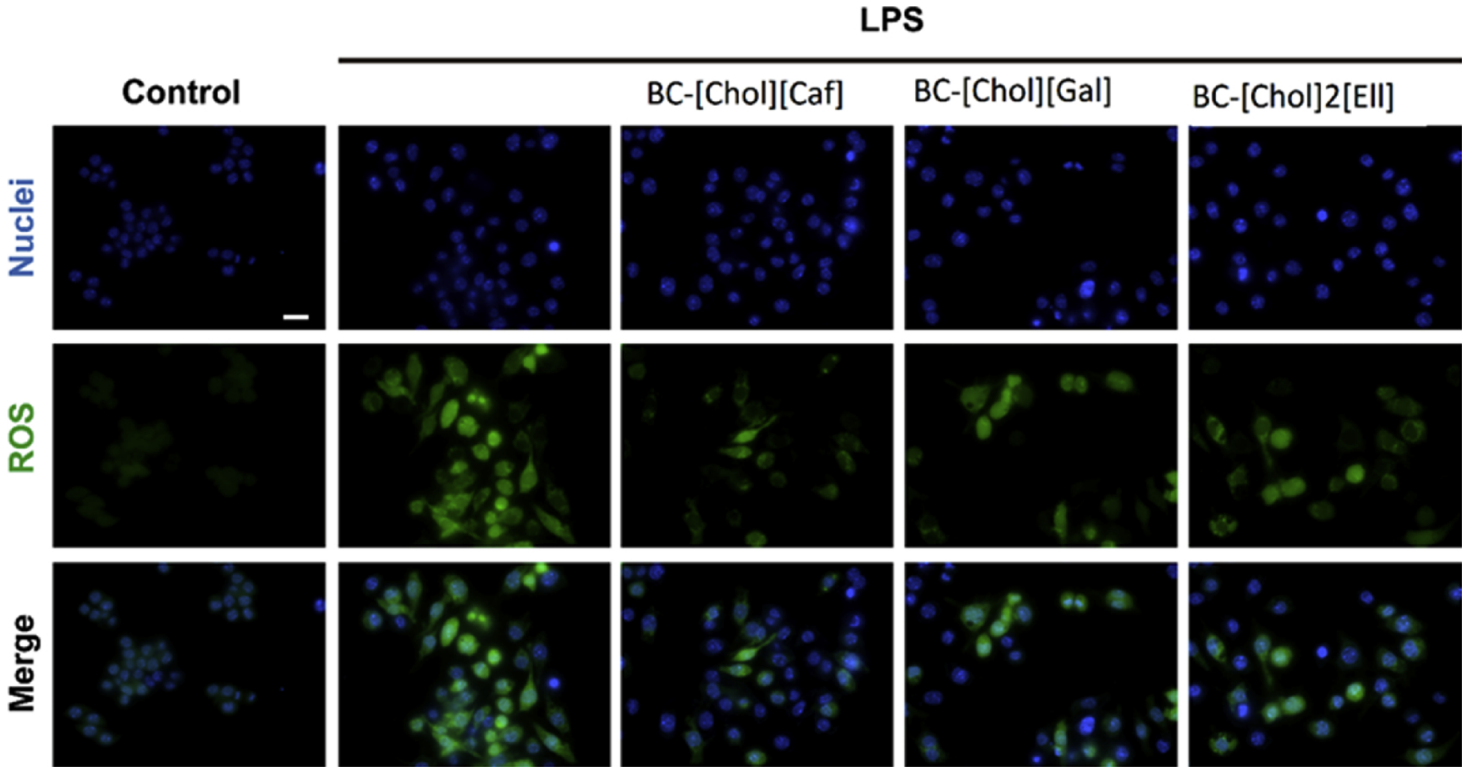

Fig. 8. Evaluation of the capacity of BC-ILs membranes to prevent LPS-induced oxidative stress in macrophages. Cells were exposed to a set of different conditions and the ROS production was assessed using $\mathrm{H}_{2}$ DCFDA probe (in green). Hoechst (blue) was used to stain the cells nucleus. Images were acquired at a magnification of $63 \times$. Scale bar: $20 \mu \mathrm{m}$. (For interpretation of the references to colour in this figure legend, the reader is referred to the web version of this article.)

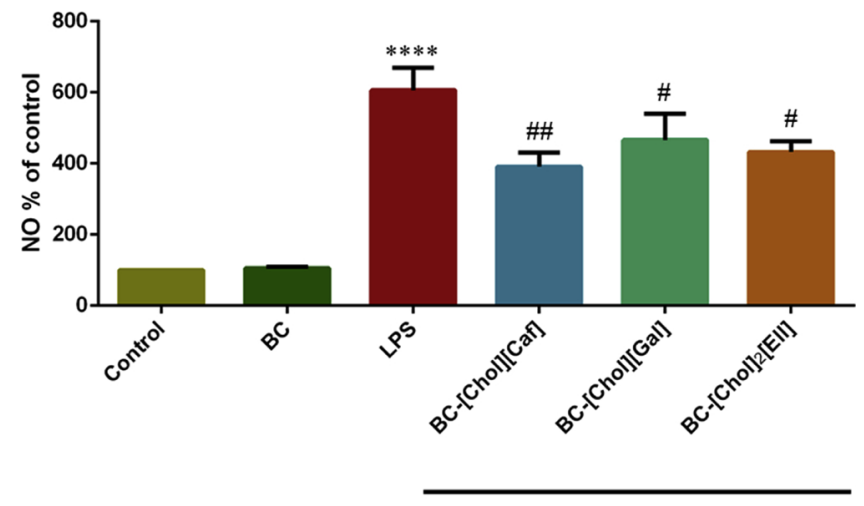

LPS

Fig. 9. Evaluation of the capacity of BC-ILs membranes to prevent LPS-induced NO production in macrophages. NO production in relation to the control $(n=3)$. Cells were treated with the different BC-ILs membranes conditioned mediums and then stimulated with LPS. The NO production was assed via a Griess assay. Data are presented as percentage of NO production in relation to control and represent the average \pm SD from at least 3 independent experiments. (****p $<0.0001$; control vs LPS; ${ }^{*} \mathrm{p}<0.05$; ${ }^{\# \#} \mathrm{p}<0.01$ : BC-ILs membranes vs LPS).

sustained release through the human skin, as shown by the skin permeation and dissolution assays here presented. Furthermore, the BC-IL membranes are non-cytotoxic, and have high antioxidant and anti-inflammatory activity that are fundamental aspects in clinical situations related with skin injury. Additionally, the outstanding properties of BC for skin treatment such as control over the amount of applied drug, allowing the use of lower amounts of product, absorption of exudates in case of damaged skin and the protection of damaged skin from external agents, reducing the risk of infections, makes this material ideal for the desired application.

In summary, the bioactive BC-IL membranes developed in this work can be safely used in human beings, for skin care and treatment (for example burned or damaged skin) with no health risks. These biomaterials are innovative and biocompatible options for the biomedical and cosmetic industries. Further studies comprising in-vivo tests should be carried out to confirm the effectiveness of the prepared membranes.

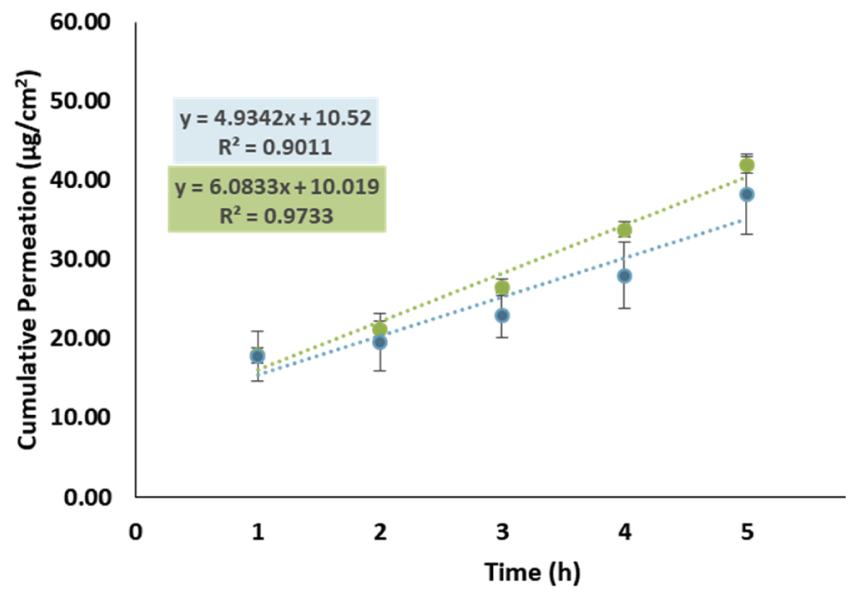

Fig. 10. Cumulative amount that permeated epidermis during the $5 \mathrm{~h}$ assay for $\mathrm{BC}-[\mathrm{Chol}][\mathrm{Gal}]$ (๑) and $\mathrm{BC}-[\mathrm{Chol}][\mathrm{Caf}]$ (๑) (results are expressed as average $\pm \mathrm{SD})$.

\section{Author contributions}

The manuscript was written through contributions of all authors. All authors have given approval to the final version of the manuscript.

\section{Funding sources}

This work was developed in the scope of the project CICECO-Aveiro Institute of Materials (Ref. FCT UID/CTM/50011/2013), financed by national funds through the FCT/MEC and co-financed by FEDER under the PT2020 Partnership Agreement.

The authors are grateful for financial support from FCT for the doctoral grant SFRH/593 BD/85871/2012 of T.E. Sintra, SFRH/BD/ $85690 / 2012$ of N.H. Silva and C. Freire and S.P.M. Ventura Researcher contracts, IF/01407/2012 and IF/00402/2015, respectively. S.A.O. Santos thanks the project AgroForWealth (CENTRO-01-0145-FEDER000001) for the financial support. M. G. Freire acknowledges the European Research Council (ERC) for the Starting Grant ERC 2013-StG337753. 


\section{Appendix A. Supplementary data}

Supplementary material related to this article can be found, in the online version, at doi:https://doi.org/10.1016/j.carbpol.2018.10.051.

\section{References}

Alam, M. N., Bristi, N. J., \& Rafiquzzaman, M. (2013). Review on in vivo and in vitro methods evaluation of antioxidant activity. Saudi Pharmaceutical Journal, 21(2), 143-152.

Barud, S., Miguel, A., Júnior, D. A., Saska, S., Mestieri, L. B., Alvares, J., et al. (2013). Antimicrobial Brazilian Propolis (EPP-AF) containing biocellulose membranes as promising biomaterial for skin wound healing. Evidence-Based Complementary and Alternative Medicine, 2013, 1-10.

Brien, J. O., Wilson, I., Orton, T., \& Pognan, Ë. (2000). Investigation of the Alamar Blue (resazurin) fluorescent dye for the assessment of mammalian cell cytotoxicity. Journal of Biochemistry, 5426, 5421-5426.

Chawla, P. R., Bajaj, I. B., Survase, S. A., \& Singhal, R. S. (2009). Microbial cellulose: Fermentative production and applications. Food Technology Biotechnology, 47(2), 107-124.

Corbett, S., Daniel, J., Drayton, R., Field, M., Steinhardt, R., \& Garrett, N. (2010). Evaluation of the anti-inflammatory effects of ellagic acid. Journal of Perianesthesia Nursing, 25(4), 214-220.

Cunha, A. G., Freire, C. S. R., Silvestre, A. J. D., Neto, C. P., Gandini, A., Orblin, E., et al. (2007). Bi-phobic cellulose fibers derivatives via surface trifluoropropanoylation. Langmuir, 9(12), 10801-10806.

Czaja, W., Krystynowicz, A., Bielecki, S., \& Brown, R. M. (2006). Microbial cellulose-The natural power to heal wounds. Biomaterials, 27(2), 145-151. https://doi.org/10. 1016/j.biomaterials.2005.07.035.

Czaja, W., Krystynowicz, A., Kawecki, M., Wysota, K., Sakiel, S., Wroblewski, P., et al. (2007). In R. M. Brown (Ed.). Cellulose: Molecular and structural biology (pp. 307-319). Springer.

Dai, J., \& Mumper, R. J. (2010). Plant phenolics: Extraction, analysis and their antioxidant and anticancer properties. Molecules (Basel, Switzerland), 15(10), 7313-7352. https://doi.org/10.3390/molecules15107313.

Drury, J. L., \& Mooney, D. J. (2003). Hydrogels for tissue engineering: Scaffold design variables and applications. Biomaterials, 24(24), 4337-4351.

Dubey, D., Malviya, R., \& Sharma, P. K. (2014). Mathematical modelling and release behaviour of drug. Drug Delivery Letters, 4, 254-268.

Erbas Kiziltas, E., Kiziltas, A., Blumentritt, M., \& Gardner, D. J. (2015). Biosynthesis of bacterial cellulose in the presence of different nanoparticles to create novel hybrid materials. Carbohydrate Polymers, 129, 148-155.

Fontana, J. D., de Souza, A. M., Fontana, C. K., Torriani, I. L., Moreschi, J. C., \& Galloti, B. J. (1990). Acetobacter cellulose pellicle as a temporary skin substitute. Applied Biochemestry and Biotechnology, 24(25), 253-254.

Guidi, I., Galimberti, D., Lonati, S., Novembrino, C., Bamonti, F., Tiriticco, M., et al. (2006). Oxidative imbalance in patients with mild cognitive impairment and Alzheimer's disease. Neurobiology of Aging, 27(2), 262-269.

Helenius, G., Bäckdahl, H., Bodin, A., Nannmark, U., Gatenholm, P., \& Risberg, B. (2006). In vivo biocompatibility of bacterial cellulose. Journal of Biomedical Materials Research Part A, 76(2), 431-438.

Hestrin, S., \& Schramm, M. (1953). Synthesis of cellulose by Acetobacter xylinum. Biochemistry Journal, 58, 345-352.

Huang, D., Ou, B., \& Prior, L. R. (2005). The chemistry behind antioxidant capacity assays. Journal of Agricultural and Food Chemistry, 53, 1841-1856.

Jeong, S., Il, Lee, S. E., Yang, H., Jin, Y.-H., Park, C.-S., et al. (2010). Toxicologic evaluation of bacterial synthesized cellulose in endothelial cells and animals. Molecular \{ \&) Cellular Toxicology, 6(4), 370-377.

Jones, I., Currie, L., \& Martin, R. (2002). A guide to biological skin substitutes. British Journal of Plastic Surgery, 55, 185-193.

Jung, W.-K., Lee, D.-Y., Kim, J.-H., Choi, I., Park, S.-G., Seo, S.-K., et al. (2008). Antiinflammatory activity of caffeic acid phenethyl ester (CAPE) extracted from Rhodiola sacra against lipopolysaccharide-induced inflammatory responses in mice. Process Biochemistry, 43(7), 783-787.

Klingman, A. M., \& Christophel, E. (1963). Preparation of isolated sheets of human stratum stratum couneum. Arch Dermatology, 88(6), 705-706.

Kono, H., Yunoki, S., Shikano, T., \& Fujiwara, M. (2002). CP/MAS 13C NMR study of cellulose and cellulose derivatives. 1. Complete assignment of the CP/MAS 13C NMR spectrum of the native cellulose. Journal of the American Chemical Society, 124(9), $7506-7511$.

Kovacic, P., \& Somanathan, R. (2013). Broad overview of oxidative stress and its complications in human health. Open Journal of Preventive Medicine, 3(1), 32-41.

Kroes, B. H., van den Berg, A. J. J., Quarles van Ufford, H. C., van Dijk, H., \& Labadie, R.
P. (1992). Anti-inflammatory activity of gallic acid. Plant Medicine, 58, 499-504.

Kusumawati, I., \& Gunawan, I. (2013). In I. Kusumawati, \& I. Gunawan (Eds.). Studies in natural products chemistry (pp. 485-505). (1st ed.). Amsterdam: Elsevier.

Ma, Y., Xin, L., Tan, H., Fan, M., Li, J., Jia, Y., et al. (2017). Chitosan membrane dressings toughened by glycerol to load antibacterial drugs for wound healing. Materials Science and Engineering C, 81(August), 522-531.

Mandal, S., Yadav, S., Yadav, S., \& Nema, R. K. (2009). Antioxidants: A review. Journal of Chemical and Pharmaceutical Research, 1(1), 102-104.

Marrucho, I. M., Branco, L. C., \& Rebelo, L. P. N. (2014). Ionic liquids in pharmaceutical applications. Annual Review of Chemical and Biomolecular Engineering, 5, 527-546.

Massaad, C. A. (2011). Neuronal and vascular oxidative stress in Alzheimer's disease. Current Neuropharmacology, 9, 662-673.

Mayall, R. C., Mayall, A. C., Mayall, L. C., Rocha, H. C., \& Marques, L. C. (1990). Tratamento das ulceras troficas dos membros com um novo substitute da pele. Revista Brasileira de Cirurgia, 80(4), 120-124.

Meshitsuka, G., \& Isogai, A. (1995). Chemical structures of cellulose, hemicelluloses, and lignin. Chemical modification of lignocellulosic materials. New York: CRC Press11-34.

Mohd Amin, M. C. I., Ahmad, N., Halib, N., \& Ahmad, I. (2012). Synthesis and characterization of thermo- and pH-responsive bacterial cellulose/acrylic acid hydrogels for drug delivery. Carbohydrate Polymers, 88(2), 465-473.

Nguyen, V. T., Gidley, M. J., \& Dykes, G. A. (2008). Potential of a nisin-containing bacterial cellulose film to inhibit Listeria monocytogenes on processed meats. Food Microbiology, 25(3), 471-478.

Plechkova, N. V., \& Seddon, K. R. (2008). Applications of ionic liquids in the chemical industry. Chemical Society Reviews, 37(1), 123-150.

Podda, M., \& Grundmann-Kollmann, M. (2001). Low molecular weight antioxidants and their role in skin ageing. Clinical Dermatology, 26, 578-582.

Qiu, Y., Qiu, L., Cui, J., \& Wei, Q. (2016). Bacterial cellulose and bacterial cellulosevaccarin membranes for wound healing. Materials Science \& Engineering. 59, 303-309.

Queimada, J. A., Mota, L. F., Pinho, P. S., \& Macedo, A. E. (2009). Solubilities of biologically active phenolic compounds: Measurements and modeling. Journal Phys Chem, 113, 3469-3476.

Rogers, R. D., \& Seddon, K. R. (2003). Ionic liquids—Solvents of the Future? Science, 302, 792-793.

Sas, K., Robotka, H., Toldi, J., \& Vécsei, L. (2007). Mitochondria, metabolic disturbances, oxidative stress and the kynurenine system, with focus on neurodegenerative disorders. Journal of the Neurological Sciences, 257(1-2), 221-239.

Schirhagl, R. (2014). Bioapplications for molecularly imprinted polymers. Analytical Chemistry, 86(1), 250-261.

Silva, N. H. C. S., Drumond, I., Almeida, I. F., Costa, P., Rosado, C. F., Neto, C. P., et al. (2013). Topical caffeine delivery using biocellulose membranes: A potential innovative system for cellulite treatment. Cellulose, 21(1), 665-674.

Silva, N. H. C. S., Rodrigues, A. F., Almeida, I. F., Costa, P. C., Rosado, C., Neto, C. P., et al. (2014). Bacterial cellulose membranes as transdermal delivery systems for diclofenac: In vitro dissolution and permeation studies. Carbohydrate Polymers, 106, 264-269.

Silver, F. H. (1994). Wound dressings and skin replacement. Biomaterials, medical devices and tissue engineering: An integrated approach. Dordrecht: Springer.

Sintra, T., Luís, A., Rocha, S., Ferreira Lobo, A., Gonçalves, F., Santos, L., et al. (2015). Enhancing the antioxidant characteristics of phenolic acids by their conversion into cholinium salts. ACS Sustainable Chemistry \& Engineering, 3(10), 2558-2565.

Tokoh, C., Takabe, K., Sugiyama, J., \& Fujita, M. (2002). CP/MAS 13 C NMR and electron diffraction study of bacterial cellulose structure affected by cell wall polysaccharides. Cellulose, 9, 351-360.

Tomé, L. C., Silva, N. H. C. S., Soares, H. R., Coroadinha, A. S., Sadocco, P., Marrucho, I. M., et al. (2015). Bioactive transparent films based on polysaccharides and choliniumcarboxylate ionic liquids. Green Chemistry, 17, 4291-4299.

Trovatti, E., Serafim, L. S., Freire, C. S. R., Silvestre, A. J. D., \& Neto, C. P. (2011). Gluconacetobacter sacchari: An efficient bacterial cellulose cell-factory. Carbohydrate Polymers, 86(3), 1417-1420.

Trovatti, E., Silva, N. H. C. S., Duarte, I. F., Rosado, C. F., Almeida, I. F., Costa, P., et al. (2011). Biocellulose membranes as supports for dermal release of lidocaine. Biomacromolecules, 12, 4162-4168.

Wei, B., Yang, G., \& Hong, F. (2011). Preparation and evaluation of a kind of bacterial cellulose dry films with antibacterial properties. Carbohydrate Polymers, 84(1), 533-538.

Wiegand, C., Elsner, P., Hipler, U., \& Klemm, D. (2006). Protease and ROS activities influenced by a composite of bacterial cellulose and collagen type I in vitro. Cellulose, 13, 689-696.

Yamanaka, S., Watanabe, K., Kitamura, N., Igushi, M., Mitsuhashi, S., Nishi, Y., et al. (1989). The structure and mechanical properties of sheets prepared from bacterial cellulose. Journal of Materials Science, 24, 1-5.

Yu, T., \& Anderson, D. (1997). Reactive oxygen species-induced DNA damage and its modification: A chemical investigation. Fundamental and Molecular Mechanisms of Mutagenesis, 379. 